\title{
Effects of quadrupole fringe fields in final focus systems for linear colliders
}

\author{
Marcin Patecki ${ }^{1,2}$ and Rogelio Tomás ${ }^{1}$ \\ ${ }^{1}$ European Organization for Nuclear Research (CERN), CH-1211 Geneva 23, Switzerland \\ ${ }^{2}$ Department of Physics, Warsaw University of Technology, Plac Politechniki 1, 00-661 Warsaw, Poland
}

(Received 20 May 2014; published 3 October 2014)

\begin{abstract}
Quadrupole fringe fields in the final focus system can be a source of aberrations in the interaction point transverse beam sizes. This paper investigates the fringe field impact on the transverse beam size in the ATF2, ILC, and CLIC lattices in the linear and non-linear regimes. The linear effects are studied by replacing the hard-edge quadrupolar field by the more realistic gradient fall-off. To address the nonlinear effects, the fringe fields are represented as high order kicks added to both sides of the hard-edge magnets. It will be shown that the linear fringe fields effects can be easily cured by tuning the quadrupole strengths. On the other hand, mitigation of the nonlinear fringe fields effects is more difficult and requires use of octupole magnets or, alternatively, increasing the value of interaction point horizontal beta function $\beta_{x}^{*}$.
\end{abstract}

DOI: 10.1103/PhysRevSTAB.17.101002

PACS numbers: 29.20.Ej, 41.85.Lc

\section{INTRODUCTION}

The final focus system (FFS) is the last [before the interaction point (IP)] section of a linear collider. Its main purpose is to squeeze the beam size at the IP to the required value. For the future linear colliders the transverse beam size should be in the order of nanometers to reach the expected luminosity. The final part of the FFS is usually formed by a final doublet (FD), which is a pair of strong quadrupole magnets located upstream from the IP. As those magnets are in high beta region, the magnetic field imperfections including the fringe fields can significantly affect the beam size.

The lattices considered are the FFS of CLIC [1,2], ILC [3] and 3 versions of the Accelerator Test Facility 2 (ATF2) $[4,5]$. See Table I for the comparison of their design parameters. The calculation of beam size is performed order by order with MAPCLASS2 [6] with the use of lattice transfer map obtained with PTC [7], see [8] for details. Magnet imperfections other than fringe fields are neglected throughout this paper. A detailed study of the magnetic imperfections impact on the beam size for ATF2 can be found in [9].

\section{LINEAR EFFECTS OF THE FRINGE FIELDS}

The hard-edge model, widely used in beam lines modeling, assumes that magnetic field changes steplike at the border of the magnet, while in reality it changes smoothly and fringes outside the magnet. A more accurate modeling of the magnetic field along the longitudinal axis can be achieved by adding a series

Published by the American Physical Society under the terms of the Creative Commons Attribution 3.0 License. Further distribution of this work must maintain attribution to the author(s) and the published article's title, journal citation, and DOI. of shorter magnets (50 in this paper) at each side with progressively lower values of normalized strength $k_{1}$, as done e.g., in [10]. The magnet strengths $k_{1}$ have to be modified in a way that the integrated strength $\int k_{1} d s$ of the magnet assembly remains the same as $k_{1} L$ of the hard-edge magnet.

The comparison of the hard-edge and fringed magnet models for CLIC, ILC, and ATF2 is given in Fig. 1. The Enge function [11], see Eq. (1), fitted to simulated magnet strength data is used to model the fringe field shape.

$$
F(z)=\frac{1}{1+\exp \left[a_{0}+a_{1}(z / D)+a_{2}(z / D)^{2}+\cdots+a_{5}(z / D)^{5}\right]},
$$

where $a_{0}, \ldots, a_{5}$ are fitting parameters and $D$ is the magnet aperture diameter, see Table II.

The magnet gradient of the CLIC final doublet normal conducting QD0 quadrupole is obtained from magnetic simulation [12] and fitted with two Enge functions (ensuring continuity), one for the region inside the magnet and a second for the outside region. This provides a good

TABLE I. Comparison between relevant parameters of the considered beam lines.

\begin{tabular}{lcccccccc}
\hline \hline & $\begin{array}{c}E \\
{[\mathrm{GeV}]}\end{array}$ & $\begin{array}{c}L^{*} \\
{[\mathrm{~m}]}\end{array}$ & $\begin{array}{c}\beta_{x}^{*} \\
{[\mathrm{~mm}]}\end{array}$ & $\begin{array}{c}\beta_{y}^{*} \\
{[\mathrm{~mm}]}\end{array}$ & $\begin{array}{c}\sigma_{x}^{*} \\
{[\mathrm{~nm}]}\end{array}$ & $\begin{array}{c}\sigma_{y}^{*} \\
{[\mathrm{~nm}]}\end{array}$ & $\begin{array}{c}\text { QD0 } k_{1} \\
{\left[\mathrm{~m}^{-2}\right]}\end{array}$ \\
\hline Project & 250 & 3.5 & 8 & 0.1 & 207 & 2.4 & -0.0772 \\
CLIC 500 GeV & 250 & 3.5 & 11 & 0.48 & 481 & 6.1 & -0.1379 \\
ILC & 1.3 & 1 & 4 & 0.1 & 2910 & 37 & -2.8715 \\
ATF2 nominal & 1.3 & 1 & 4 & 0.025 & 3300 & 23 & -2.8751 \\
ATF2 ultra low $\beta^{*}$ & 1.3 & & & \\
ATF2 ultra low $\beta^{*}$, & 1.3 & 1 & 40 & 0.025 & 9000 & 23 & -2.8667 \\
$\quad 10 \beta_{x}^{*}$ & & & & & & & \\
\hline \hline
\end{tabular}



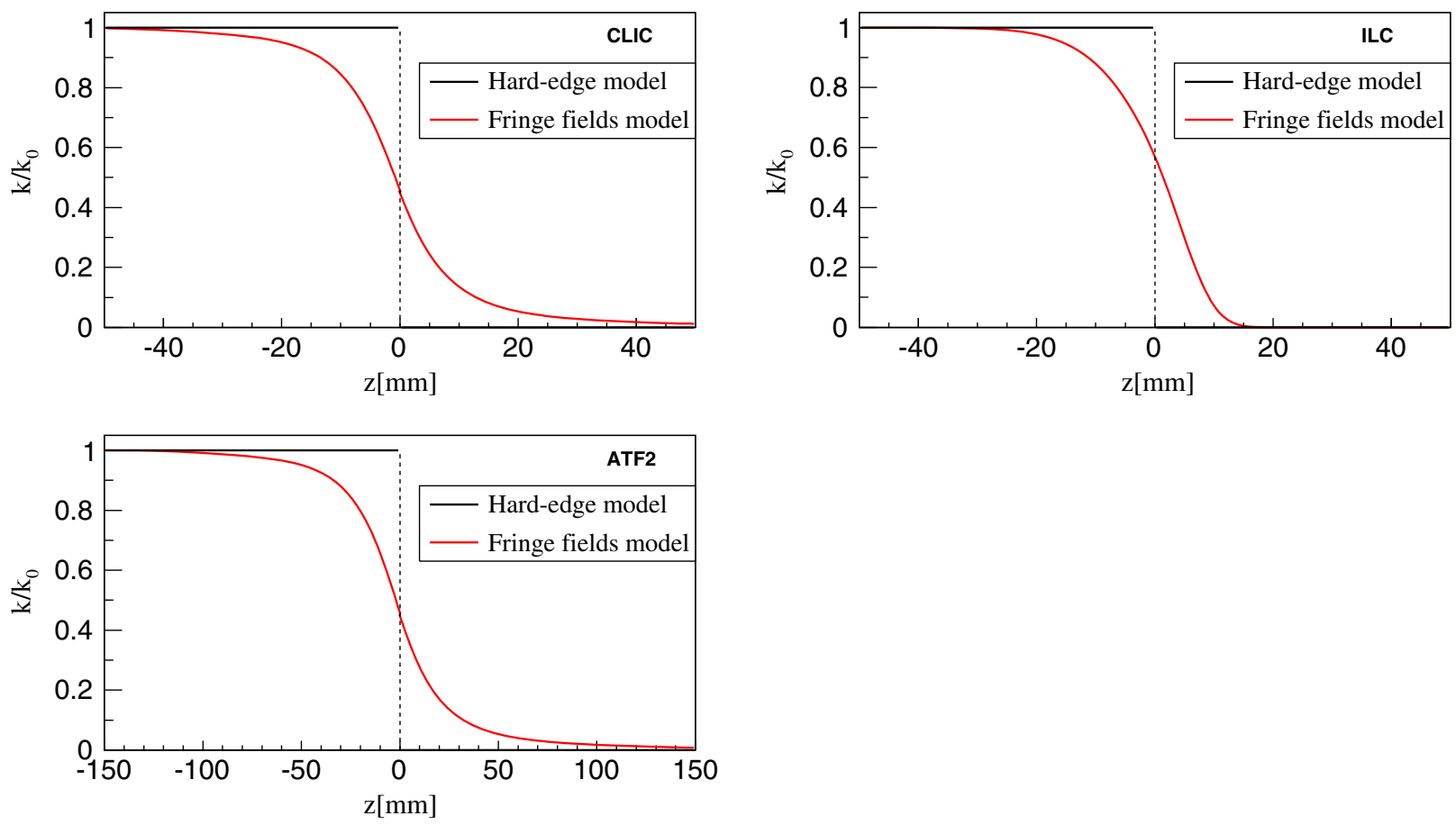

FIG. 1. Hard-edge and fringe fields models. Note the different longitudinal scale for each case, which depends on the aperture.

fringe shape description, as it turns out that using just one Enge function for the whole fringe region is not sufficient. The fit parameters and magnets apertures are given in Table II. Magnetic measurements or simulations of ATF2 quadrupoles were not available so the fringe region was modeled with the CLIC quadrupole parameters scaled with the aperture according to Eq. (1). The ILC quadrupoles are planned to be super conducting so the fitting parameters of super conducting magnet are needed. As the ILC magnetic simulation was not available, the HL-LHC MQXF magnets [13] data were used applying the corresponding aperture scaling. The apertures for final doublet quadrupoles are $20 \mathrm{~mm}$ for CLIC and ILC, and $50 \mathrm{~mm}$ for ATF2.

\section{A. CLIC and ILC BDS lattices}

Figures 2 and 3 present the impact of the linear fringe fields of the final doublet magnets on the transverse

TABLE II. Parameters of the fringe field model.

\begin{tabular}{lccc}
\hline \hline Parameter & CLIC QD0 $(z<0)$ & CLIC QD0 $(z>0)$ & MQXF \\
\hline$a_{0}$ & 0.166 & 0.205 & -0.283 \\
$a_{1}$ & -4.313 & -4.127 & -3.836 \\
$a_{2}$ & 1.170 & -1.830 & 1.948 \\
$a_{3}$ & 0.030 & -0.340 & -2.697 \\
$a_{4}$ & -0.003 & 0.057 & 1.289 \\
$a_{5}$ & -0.007 & 0.029 & -0.186 \\
$\mathrm{D}[\mathrm{mm}]$ & 20 & 20 & 150 \\
\hline \hline
\end{tabular}

beam size for CLIC and ILC. The change of the IP transverse beam size due to the liner fringe field model is small (maximum beam size change is about 2\%) and can be easily corrected by adjusting the FD quadrupoles strengths.

\section{B. ATF2 lattices}

For ATF2 lattices, the beam size is more sensitive to the linear fringe fields which is probably caused by two factors. First, the ATF2 FD fringe fields region is larger, because of the larger magnet apertures. The second reason is that the normalized gradient of the ATF2 FD magnets is significantly higher than in the case of CLIC and ILC (see Table I), which enhances the fringe field effects. As it is shown in Figs. 4 and 5, one order of magnitude increase of the IP transverse beam size is observed due to the linear fringe fields model. However, it is possible to recover the original beam sizes, as also shown in the Figs. 4 and 5, by changing the FD quadrupole gradients. The new gradients are compared to the design values in Table III. The experimental verification of the linear fringe fields mitigation due to FD quadrupoles tuning is difficult to perform. The reason is that in experiment the fringe fields are not split into the linear and nonlinear part. Also tuning is rather empirical procedure where many aberrations are mixed together and difficult to distinguish. Finally, ATF2 is currently running in the $10 \beta_{x}^{*}$ optic mode which is less sensitive to the fringe fields impact. 

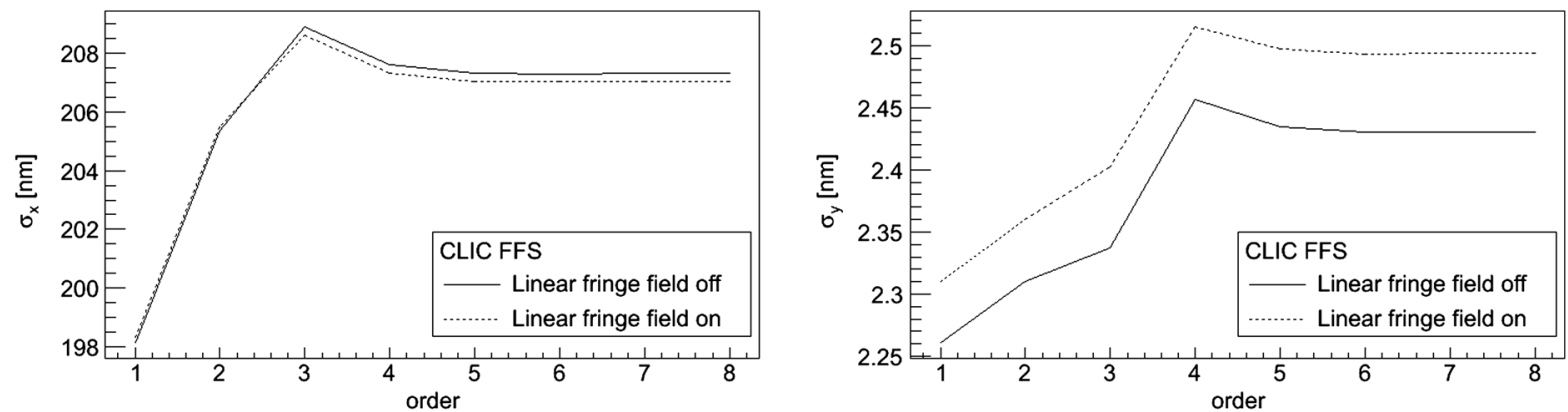

FIG. 2. Linear fringe field impact on the horizontal (left) and vertical (right) beam size in the CLIC BDS lattice. The horizontal axis stands for the polynomial order of the transfer map.
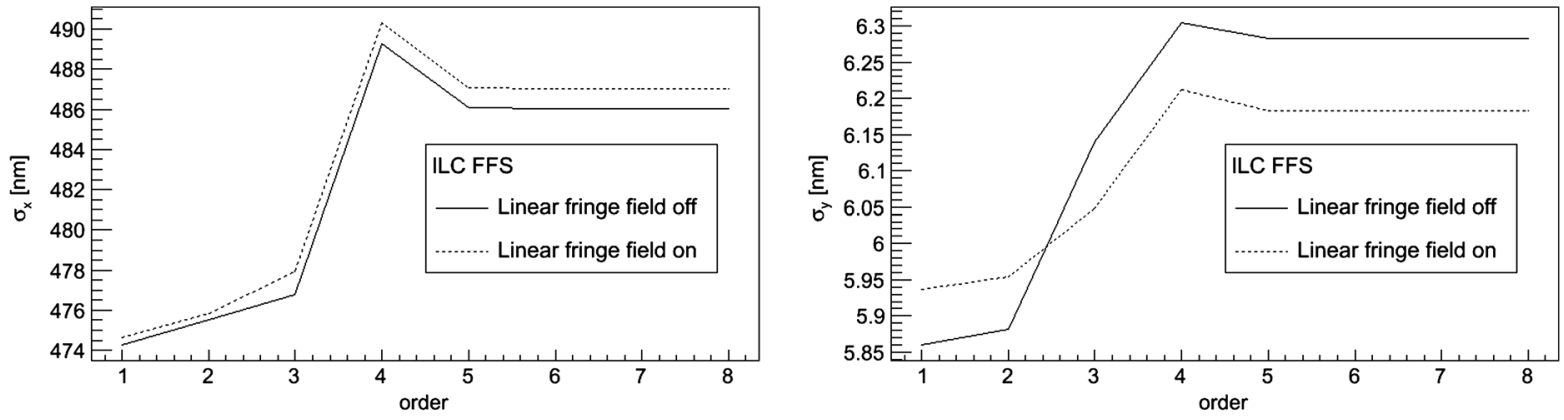

FIG. 3. Linear fringe field impact on the horizontal (left) and vertical (right) beam size in the ILC BDS lattice.
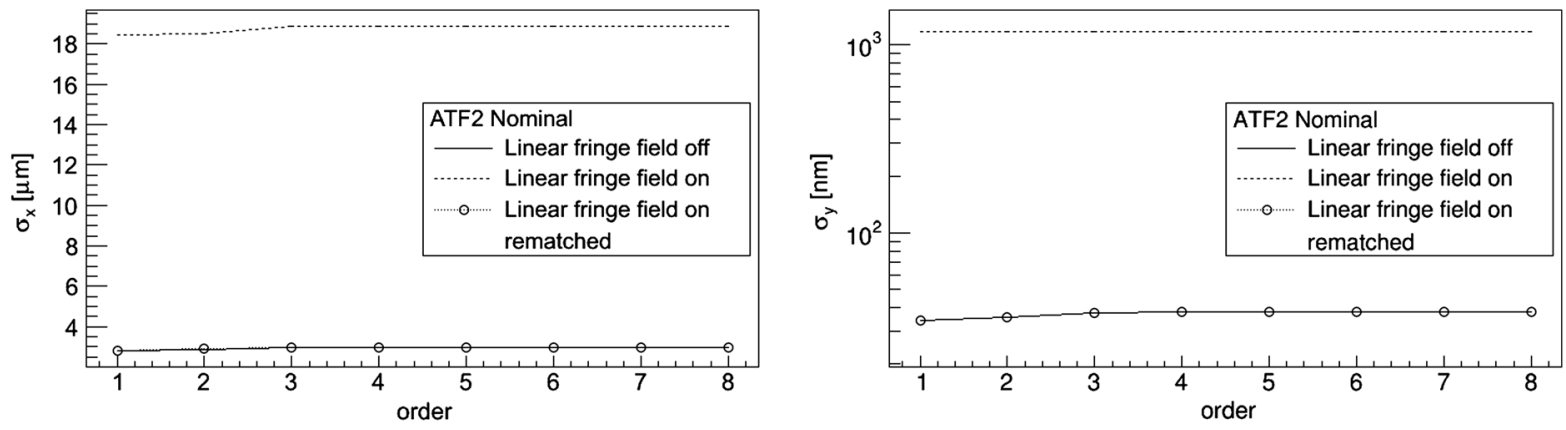

FIG. 4. Linear fringe field impact on the horizontal (left) and vertical (right) beam size in the ATF2 Nominal lattice, together with a correction of the fringe field effect using the FD quadrupoles as reported in Table III.
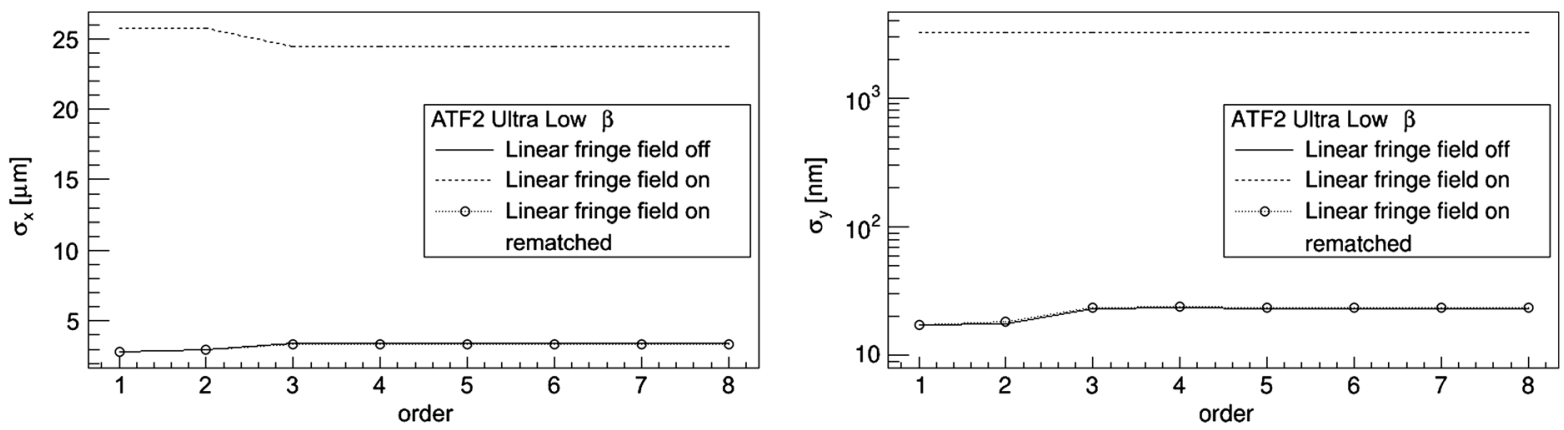

FIG. 5. Linear fringe field impact on the horizontal (left) and vertical (right) beam size in the ATF2 Ultra Low $\beta^{*}$ lattice, together with a correction of the fringe field effect using the FD quadrupoles as reported in Table III. 
TABLE III. Change of the ATF2 FD magnets strength needed to correct the linear effect of the fringe fields.

\begin{tabular}{lrrc}
\hline \hline Magnet & Design $k_{1}\left[\mathrm{~m}^{-2}\right]$ & New $k_{1}\left[\mathrm{~m}^{-2}\right]$ & Change in $\%$ \\
\hline QF1 Nominal & 1.561659 & 1.568471 & 0.436 \\
QD0 Nominal & -2.871512 & -2.886853 & 0.534 \\
QF1 Ultra Low $\beta^{*}$ & 1.566149 & 1.574011 & 0.502 \\
QD0 Ultra Low $\beta^{*}$ & -2.875086 & -2.892758 & 0.6146 \\
\hline \hline
\end{tabular}

\section{NONLINEAR EFFECTS OF THE FRINGE FIELDS}

The dynamics of the beam in the presence of nonlinear fringe field components was already studied in analytical ways [14-17] and by numerical simulation based on the magnetic field data, e.g., [18]. We approximate the fringe fields by symplectic kicks [see Eqs. (2)] applied to a particle when it enters and leaves a magnet $[19,20]$. The transfer map of the beam line including the fringe fields is computed with the use of PTC [7], and the final beam size and lattice optimization is performed with MAPCLASS2 [6]. The kicks at the magnet entrance are given by Eqs. (2).

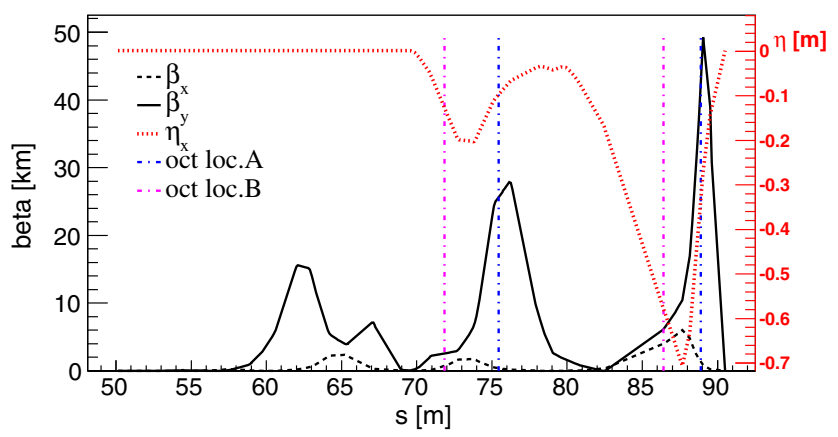

FIG. 6. $\quad \beta$ functions and dispersion along the FF line with the two octupoles locations under consideration.

$$
\begin{aligned}
\Delta x & =\frac{k_{1}}{12}\left(x^{3}+3 x y^{2}\right), \\
\Delta p_{x} & =-\frac{k_{1}}{4}\left[\left(x^{2}+y^{2}\right) p_{x}-2 x y p_{y}\right], \\
\Delta y & =-\frac{k_{1}}{12}\left(3 x^{2} y+y^{3}\right), \\
\Delta p_{y} & =\frac{k_{1}}{4}\left[\left(x^{2}+y^{2}\right) p_{y}-2 x y p_{x}\right],
\end{aligned}
$$

where $k_{1}, x, y, p_{x}, p_{y}$ are the quadrupole strength, horizontal and vertical particle transverse positions and momenta, respectively. The kicks at the exit are opposite in sign.

For CLIC and ILC FFS the nonlinear fringe fields do not affect the beam size, see Figs. 9 and 10. However, a significant effect is observed for the ATF2 lattices, see Figs. 11 and 12. The reason is that, as seen in Eqs. (2), the fringe field kicks scale with the normalized magnet gradient which has larger value for ATF2 than for CLIC and ILC, see Table I. In the case of the nominal lattice fringe fields decrease the beam size at the IP by few percent, which shows the focusing impact of the fringe fields in this particular case. However, we consider error free lattice, so this can change after applying the magnetic errors. On the other hand, for the Ultra Low $\beta^{*}$ the IP beam size increases significantly ( $\sigma_{y}^{*}$ by $47 \%$ ) being the third order the main source, as expected from Eqs. (2).

The insertion of the two octupole magnets is a proposed solution for the mitigation of the fringe fields impact on the IP beam size as they give the third order contribution to the beam dynamics. It has been already demonstrated in [9] that the octupoles can be used for the suppression of the aberrations coming from the magnetic imperfections in ATF2 beam line. The choice of the octupoles location depends on the Twiss parameters and it is desired to install the octupoles in the region of high $\beta$ function. The two possible locations were investigated, marked in Fig. 7. Location A assumes the octupoles to be installed in the regions

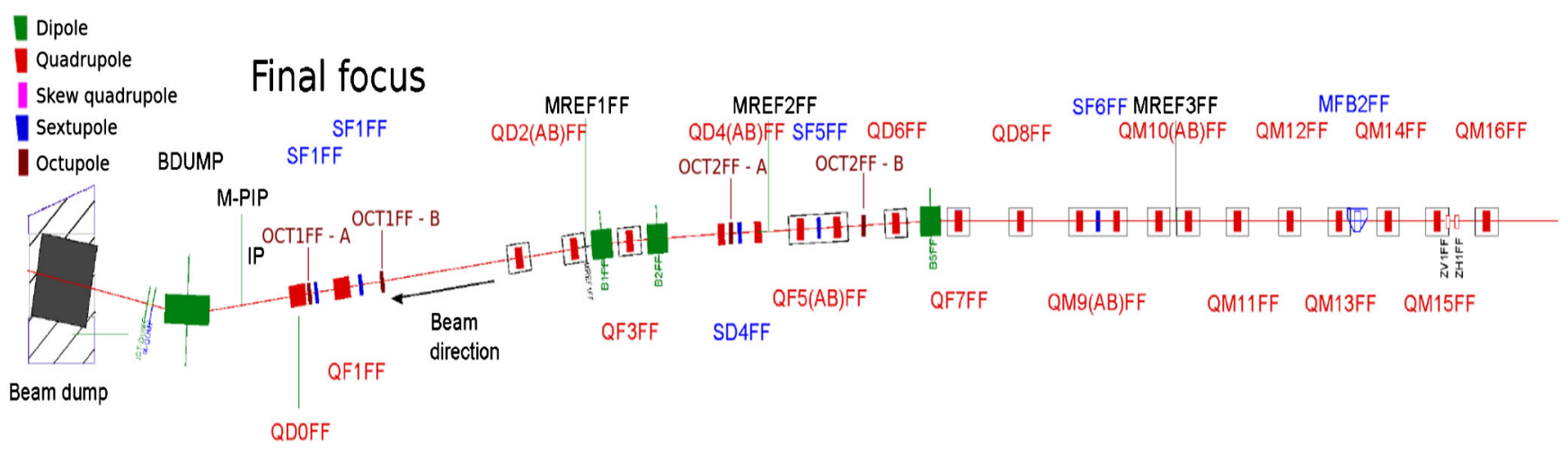

FIG. 7. Layout of ATF2 final focus line, with the two octupoles locations under consideration. 
TABLE IV. Integrated strengths of the octupole magnets in locations A and B and corresponding vertical beam size at IP of 5th order.

\begin{tabular}{lcc}
\hline \hline & Location A & Location B \\
\hline OCT1FF k $\mathrm{k}_{3}\left[\mathrm{~m}^{-3}\right]$ & -20.6 & -3.7 \\
OCT2FF k $2 \mathrm{~L}\left[\mathrm{~m}^{-3}\right]$ & 145.4 & 393.2 \\
$\sigma_{y}^{*(5)}[\mathrm{nm}]$ & 19 & 23 \\
\hline \hline
\end{tabular}

of the maximum $\beta_{y}$ function. However, this location suffers from space limitations. Therefore, location B was proposed which is less effective, but without the space constraints. The considered locations together with the ATF2 optics are given on Fig. 6. From the simulations it turns out that the location $\mathrm{A}$ is better as it significantly reduces the fringe fields aberrations (see Fig. 12) even for relatively low strength, see Table IV. The octupoles in the location B need to be much stronger, but they are feasible and sufficiently suppress the fringe fields aberrations. The vertical phase space distributions in IP for the case of the ultra low $\beta^{*}$ lattice are depicted in Fig. 8. It is clearly visible that the phase space for the case when fringe fields are included, but without octupoles, suffers from the aberrations, which can be suppressed with the use of octupoles.
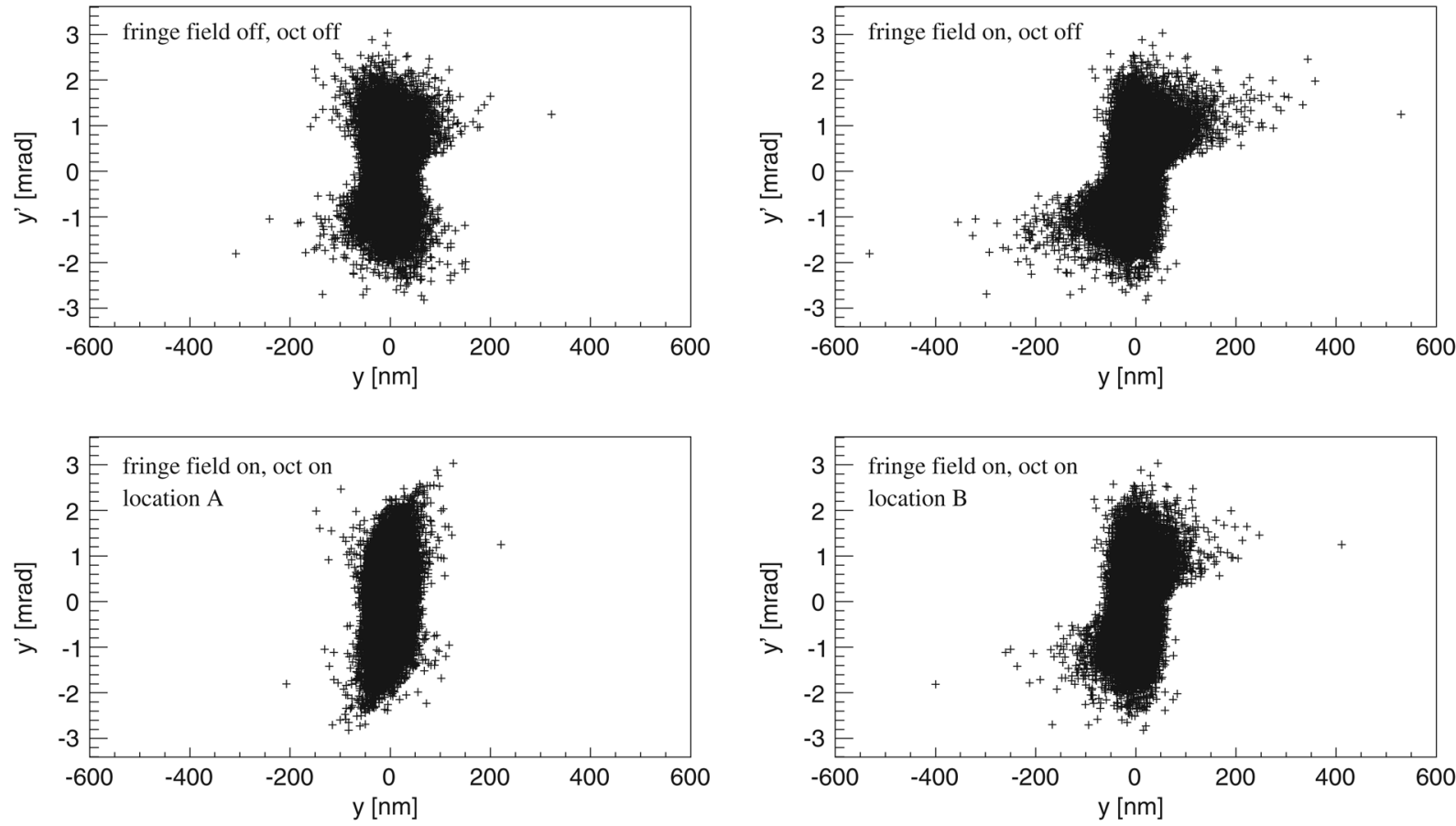

FIG. 8. The vertical phase space distributions in IP for the case of the ultra low $\beta^{*}$ lattice. The upper left plot corresponds to the case without fringe fields and without octupoles. The upper right plot corresponds to the case with fringe fields but without octupoles. Both bottom plots correspond to the case with fringe fields and octupoles included.
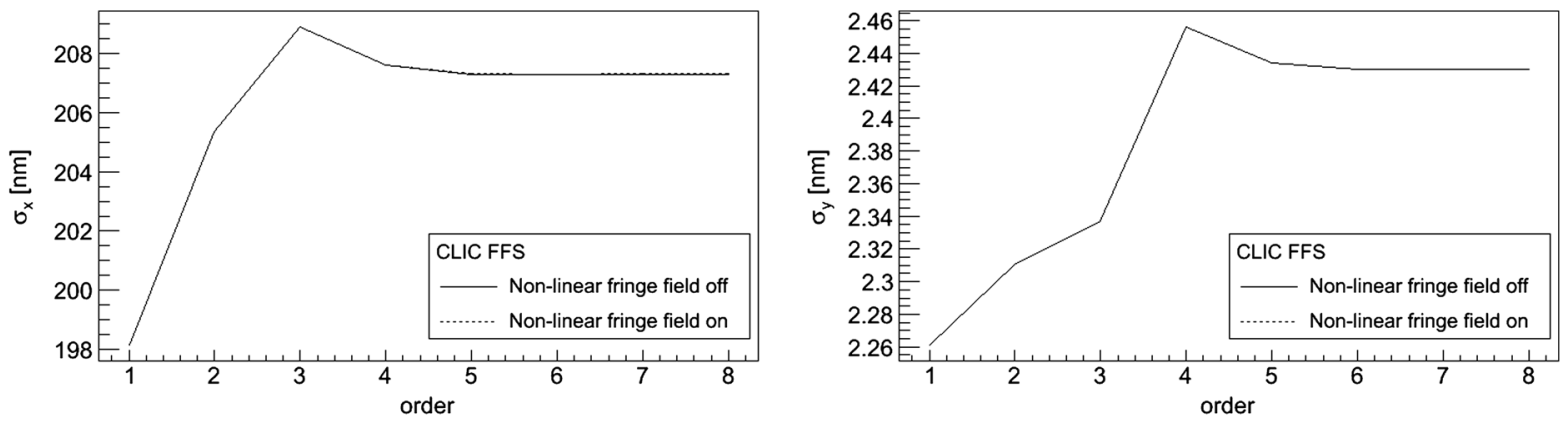

FIG. 9. Nonlinear fringe field impact on the horizontal (left) and vertical (right) beam size in the CLIC BDS lattice. 

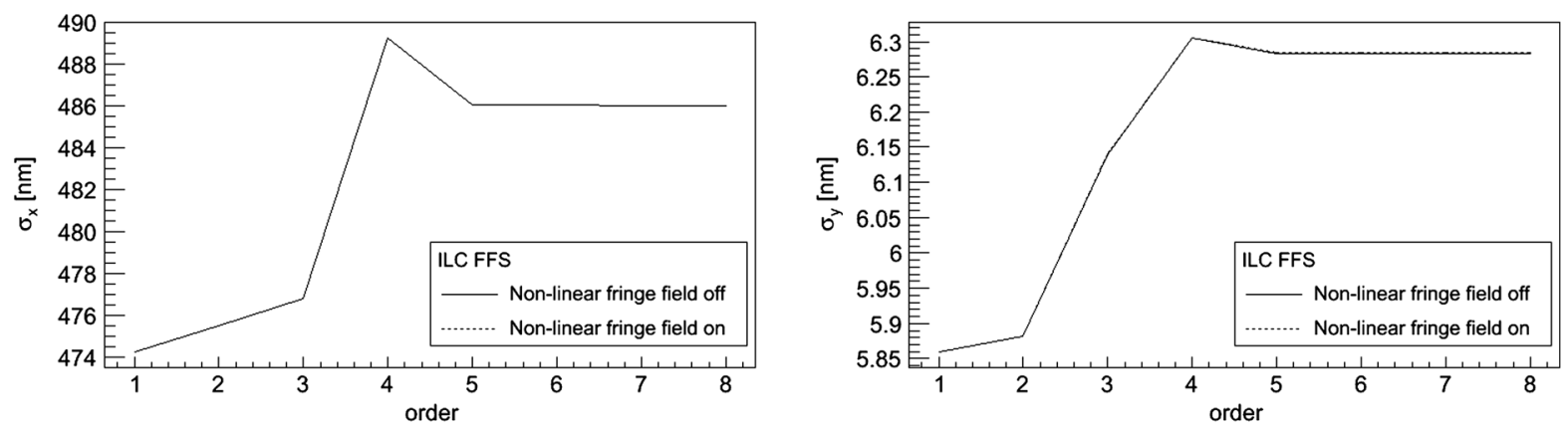

FIG. 10. Nonlinear fringe field impact on the horizontal (left) and vertical (right) beam size in the ILC BDS lattice.
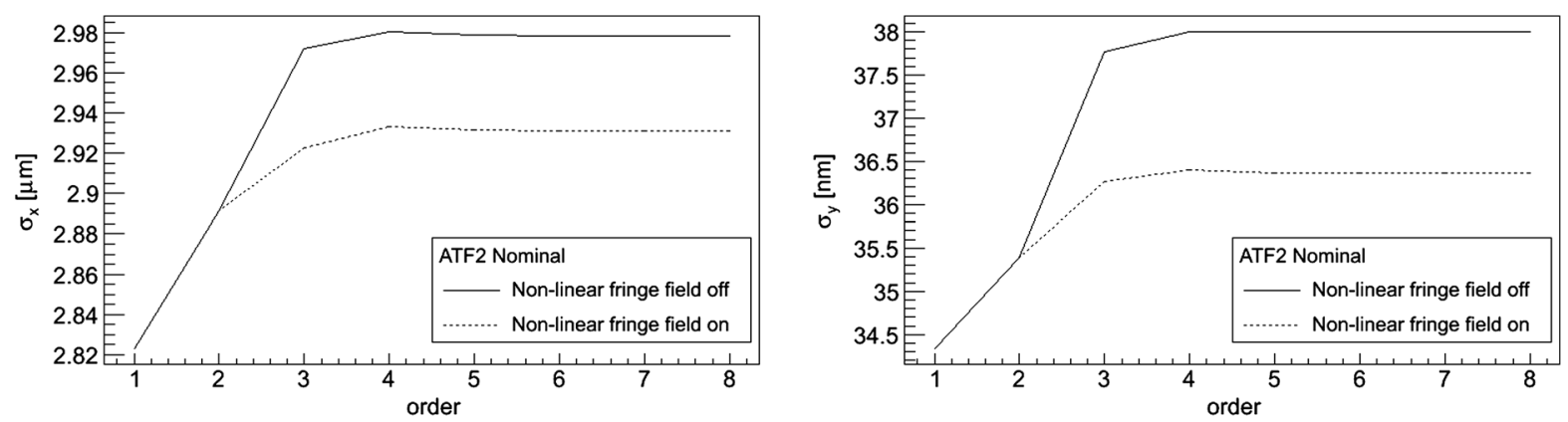

FIG. 11. Nonlinear fringe field impact on the horizontal (left) and vertical (right) beam size in the ATF2 nominal lattice.
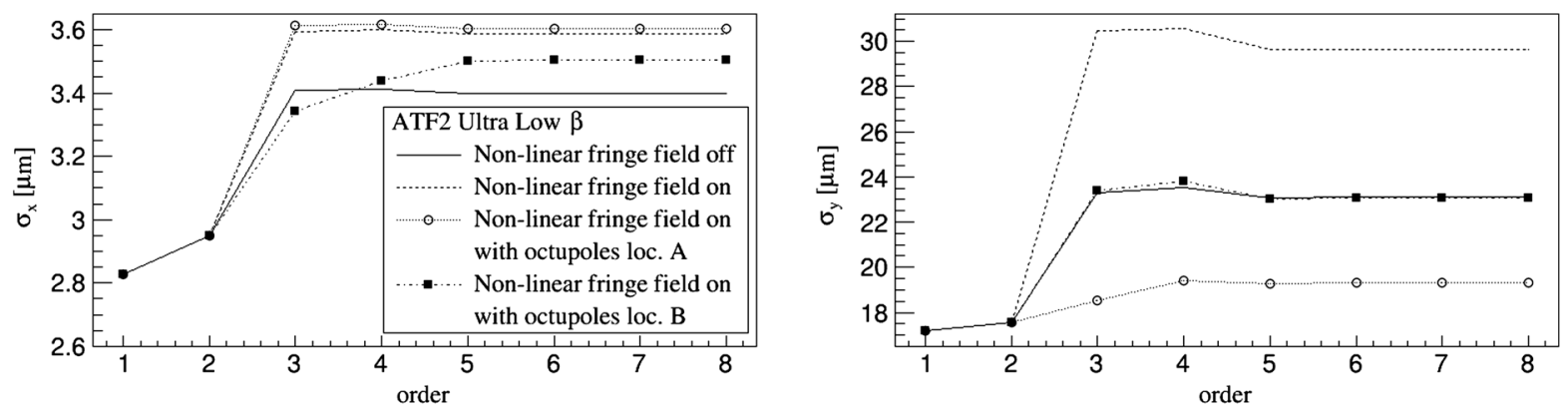

FIG. 12. Nonlinear fringe field impact on the horizontal (left) and vertical (right) beam size in the ATF2 ultra low $\beta^{*}$ lattice.
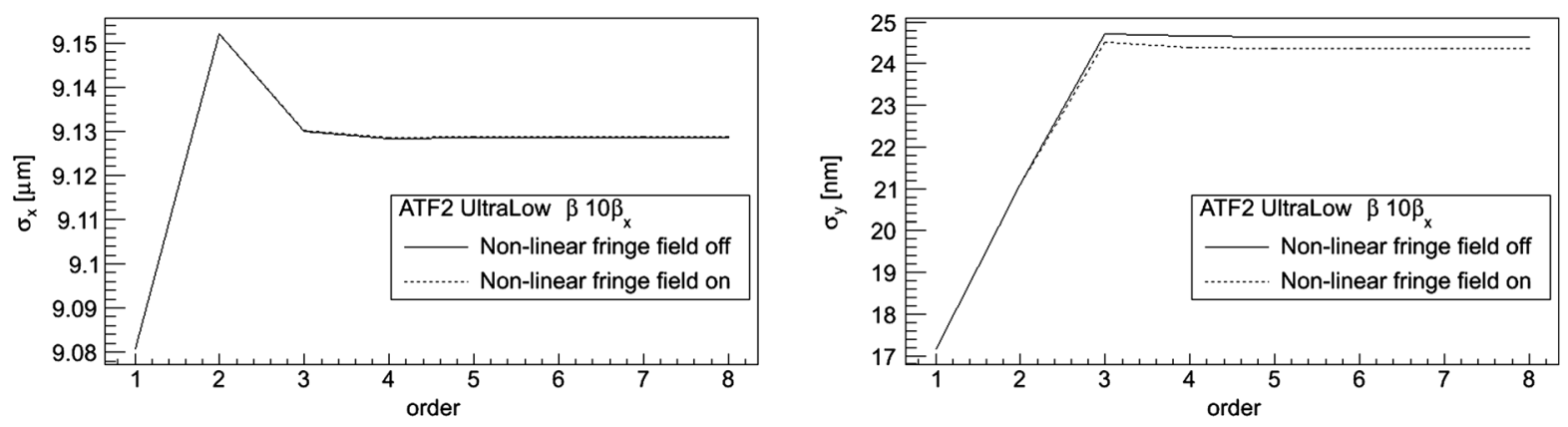

FIG. 13. Nonlinear fringe field impact on the horizontal (left) and vertical (right) beam size in the ATF2 $10 \beta_{\mathrm{x}}$ lattice. 
Another possibility (also explored in [9]) for the fringe fields effect mitigation is the increase of $\beta_{x}^{*}$, as can be seen in Fig. 13. The reason is that a higher value of $\beta_{x}^{*}$ causes the decrease of $\beta_{x}$ in final doublet and therefore the fringe fields effect is weaker. The increase of $\beta_{x}^{*}$ from $4 \mathrm{~mm}$ to $40 \mathrm{~mm}$ causes the fringe fields to be negligible.

\section{CONCLUSIONS}

Magnetic fringe fields give an unavoidable contribution to the particle transport through a beam line which can be a source of aberrations, especially for magnets in high $\beta$ regions. Understanding their impact and mitigation methods is therefore fundamental in order to avoid unwanted beam size growth.

The linearly modeled fringe fields cause a negligible increase of the transverse beam size in CLIC and ILC FFS lattices. On the other hand, for ATF2 lattices the beam size increase is significant and a correction of the final doublet magnet strength is needed.

The nonlinear model of fringe fields results in third order aberrations which are negligibly small for CLIC and ILC FFS, but are important for ATF2 ultra low $\beta^{*}$ and require corrections. The same two methods proposed in [9] to mitigate multipolar errors are verified to correct the fringe fields aberrations. The first method uses the two octupole magnets to cancel the nonlinear aberrations. The second method consists of increasing $\beta_{x}^{*}$ to reduce the effect of fringe fields in the FD.

\section{ACKNOWLEDGMENTS}

Many thanks to O. Blanco and H. Garcia for their help with MAPCLASS2 and MADX, E. Marin for providing the ATF2 lattices and fruitful discussions. Many thanks also to A. Langner for helping with the linear model implementation and to E. Todesco (MQFX) and M. Modena, A. Aloev (QD0) for the fringe field magnetic data.
[1] CLIC Conceptual Design Report, CERN-2012-007.

[2] R. Tomás, Phys. Rev. ST Accel. Beams 13, 014801 (2010).

[3] ILC Global Design Effort, ILC Technical Design Report, http://www.linearcollider.org/ILC/Publications/TechnicalDesign-Report.

[4] H. Braun et al., ATF2 Proposal. Vol. 1, CLIC-Note-636, 2005.

[5] E. Marin et al., in Proceedings of the International Particle Accelerator Conference, Kyoto, Japan (ICR, Kyoto, 2010).

[6] D. Martinez, A. Rosam, R. Tomás, and R. De Maria, Report No. CERN-ATS-Note-2012-087 TECH.

[7] F. Schmidt, E. Forest, and E. McIntosh, Report No. CERNSL-2002-044-AP.

[8] R. Tomás, Phys. Rev. ST Accel. Beams 9, 081001 (2006).

[9] E. Marin et al., Phys. Rev. ST Accel. Beams 17, 021002 (2014).

[10] S. Kelly et al., in Proceedings of the 4th International Particle Accelerator Conference, IPAC-2013, Shanghai, China, 2013 (JACoW, Shanghai, China, 2013).

[11] H. A. Enge, Rev. Sci. Instrum. 35, 278 (1964).

[12] M. Modena, arXiv:1202.5952.

[13] HiLumi LHC Work Package 3, http://espace.cern.ch/ HiLumi/WP3/SitePages/MQXF.aspx.

[14] J. Irwin and C.X. Wang, Proceedings of the Particle Accelerator Conference, Dallas, TX, 1995 (IEEE, New York, 1995).

[15] B. D. Muratori, J. K. Jones, and A. Wolski, arXiv: 1404.1762.

[16] K. Oide and H. Koiso, Phys. Rev. E 47, 2010 (1993).

[17] A. Wolski, J. Gratus, and R. Tucker, arXiv:1206.6654.

[18] M. Venturini and A. Dragt, Nucl. Instrum. Methods Phys. Res., Sect. A 427, 387 (1999).

[19] R. Baartman, "Intrinisic Third Order Aberration in Electrostatic and Magnetic Quadrupoles", Proceedings of the Particle Accelerator Conference, Vancouver, Canada, 1997.

[20] G. Hoffstatter and M. Berz, Phys. Rev. E 54, 5664, 1996. 